\title{
Remarques sur un théorème de G. Halász et A. Sárközy
}

Bulletin de la S. M. F., tome 117, no 4 (1989), p. 389-413

<http://www.numdam.org/item?id=BSMF_1989_117_4_389_0>

(C) Bulletin de la S. M. F., 1989, tous droits réservés.

L'accès aux archives de la revue «Bulletin de la S. M. F. » (http: //smf.emath.fr/Publications/Bulletin/Presentation.html) implique l'accord avec les conditions générales d'utilisation (http://www.numdam.org/ conditions). Toute utilisation commerciale ou impression systématique est constitutive d'une infraction pénale. Toute copie ou impression de ce fichier doit contenir la présente mention de copyright.

\section{Numdam}


Bull. Soc. math. France,

117, 1989, p. 389-413.

\title{
REMARQUES SUR UN THÉORÈME DE G. HALÁSZ ET A. SÁRKÖZY
}

PAR

\author{
Michel BALAZARD $(*)$
}

RÉSUMÉ. - $E$ désigne un ensemble de nombres premiers et $\Omega_{E}(n)$ le nombre de facteurs premiers de $n$ appartenant à $E$, chacun étant compté avec sa multiplicité. On donne un encadrement uniforme du nombre des entiers $n \leq x$ vérifiant $\Omega_{E}(n)=k$, étendant un résultat de G. HALÁsZ et A. SÁRKÖZY.

ABstract. - $E$ stands for a set of prime numbers, and $\Omega_{E}(n)$ for the number of prime factors of $n$ lying in $E$, each counted according to its multiplicity. We give uniform lower and upper bounds for the number of integers $n \leq x$ such that $\Omega_{E}(n)=k$, thus extending a result of G. HALÁsz and A. SÁRKÖZY.

\section{Introduction et énoncé des résultats}

L'un des buts de la théorie probabiliste des nombres est de dégager, pour des fonctions définies de manière purement arithmétique, des lois de répartition simples. Ainsi, il est bien connu que le nombre de facteurs premiers d'un entier aléatoire suit approximativement une loi de Poisson. Plus précisément, posons :

$$
\Omega(n)=\sum_{p^{\nu} \| n} \nu, \quad n \text { entier } \geq 1
$$

Ainsi, $\Omega(n)$ est le nombre de facteurs premiers de l'entier $n$, comptés avec leurs multiplicités. Soit $P_{x}$ la probabilité uniforme sur l'ensemble des entiers positifs et $\leq x$. Nous avons :

$$
\begin{aligned}
& P_{x}(\Omega(n)=k) \\
& \quad=\frac{1}{\log x} F\left(\frac{k}{\log \log x}\right) \frac{(\log \log x)^{k-1}}{(k-1) !}\left(1+\mathrm{O}_{\varepsilon}\left(\frac{1}{\log \log x}\right)\right)
\end{aligned}
$$

$\left(^{*}\right)$ Texte reçu le 23 juin 1988 , révisé le 26 juin 1989

M. BAlazard, Univ. de Limoges, Département de Mathématiques, 123 av. Albert Thomas, 87060 Limoges Cedex, France. 
uniformément pour $x \geq 3$ et $1 \leq k \leq(2-\varepsilon) \log \log x(0<\varepsilon<1)$, avec

$$
F(z)=\frac{1}{\Gamma(z+1)} \prod_{p}\left(1-\frac{1}{p}\right)^{z}\left(1-\frac{z}{p}\right)^{-1} .
$$

C'est un résultat dû à L. G. SAthe (cf. [19]). Lorsque l'entier $n$ décrit l'intervalle $[1, x], \Omega(n)$ prend toute valeur entière entre 0 et $\log x / \log 2$. La fréquence d'apparition des grandes valeurs de $\Omega(n)$ a été estimée par J.-L. NicOLAS ( $c f .[12])$ :

$$
P_{x}(\Omega(n)=k)=C 2^{-k} \log \frac{x}{2^{k}}+O_{\varepsilon}\left(2^{-k} \log ^{b}\left(\frac{3 x}{2^{k}}\right)\right)
$$

uniformément pour $x \geq 3$ et $(2+\varepsilon) \log \log x \leq k \leq \log x / \log 2(0<\varepsilon)$, avec

$$
\left.C=\frac{1}{4} \prod_{p \geq 3}\left(1+\frac{1}{p(p-2)}\right) \quad \text { et } \quad \mathrm{b}=\mathrm{b}(\varepsilon) \in\right] 0,1[\text {. }
$$

Il faut noter la différence de comportement entre (1) et (2) : dans (2), la loi est essentiellement géométrique de raison $\frac{1}{2}$.

Le souci de généraliser ces résultats à une classe importante de fonctions additives a conduit différents auteurs à considérer la fonction

$$
\Omega_{E}(n)=\sum_{p^{\nu} \| n, p \in E} \nu
$$

où $E$ est un ensemble quelconque de nombres premiers. Dans ce qui suit, nous noterons $p_{1}<p_{2}<\cdots$ la suite des éléments de $E$, et $E(x)=$ $\sum_{p \leq x, p \in E}(1 / p)$. Le théorème suivant, dû à G. HALÁsz et A. SÁrközY (cf. [7] et [18]) compare la loi locale de $\Omega_{E}(n)$, pour $1 \leq n \leq x$, avec la loi de Poisson de paramètre $E(x)$ (rappelons que $E(x)=\log \log x+\mathrm{O}(1)$ si $E$ est l'ensemble de tous les nombres premiers).

ThÉorème A. - Soit $\delta \in] 0,1[$. On $a$ :

$$
P_{x}\left(\Omega_{E}(n)=k\right) \ll_{\delta} e^{-E(x)} \frac{E(x)^{k}}{k !}
$$

pour tout $E$, tout $x \geq 1$ et tout entier naturel $k$ tels que $k+1 \leq(2-\delta) E(x)$;

$$
P_{x}\left(\Omega_{E}(n)=k\right) \gg_{\delta} e^{-E(x)} \frac{E(x)^{k-1}}{(k-1) !}
$$

TOME $117-1989-\mathrm{N}^{\circ} 4$ 
pour tout $E$, tout $x \geq 1$ et tout entier $k$ tels que $1 \leq k \leq(2-\delta) E(x)$, $E(x) \geq c(\delta)$, où $c(\delta)$ est une constante positive ne dépendant que de $\delta$.

Nous ferons quatre remarques concernant ce théorème.

1) L'énoncé (4) n'est pas exactement celui donné par A. SÁrKözY dans [18] (il suppose $k \geq \delta E(x)$ ) mais il est facile de voir que sa démonstration donne bien (4).

2) Le décalage d'exposant qu'on observe entre (3) et (4) est inévitable comme le montrent (1) et, par exemple, [3]. Il est dû au fait qu'on ne peut pas prévoir l'ordre de grandeur de $P_{x}\left(\Omega_{E}(n)=0\right)$ en ne connaissant que $E(x)$, indépendamment de la distribution des nombres premiers $p \in E$, $p \leq x:$ c'est le problème du petit crible ( $c f .[5]$ et [9]).

3) Un aspect important du THÉORĖME A est son effectivité. Les constantes impliquées par les notations de Vinogradov $\ll_{\delta}$ et $\gg_{\delta}$ ne dépendent que de $\delta$ : en particulier l'entier naturel $k$ et l'ensemble $E$ peuvent dépendre de $x$. C'est pourquoi ce théorème et ses variantes sont des outils précieux de la théorie analytique des nombres, notamment dans l'étude fine des diviseurs ( $c f$. l'ouvrage récent [8]).

4) Deux formules asymptotiques précisant (3) et (4) ont été établies par G. HALÁsz. La première, effective quand $k \sim E(x)$, est l'objet de l'article [6]. La seconde est citée à la fin du chapitre 21 de [4] et fournit un équivalent de $P_{x}\left(\Omega_{E}(n)=k\right)$ si $\delta E(x) \leq k \leq(2-\delta) E(x)$ et $E(x) \rightarrow+\infty$ (cf. également [1]).

En ce qui concerne la fréquence d'apparition des grandes valeurs de $\Omega_{E}(n)$ (quand $1 \leq n \leq x, \Omega_{E}(n)$ prend toute valeur entière entre 0 et $\left.\log x / \log p_{1}\right)$ on dispose du résultat suivant, dû à K. K. NorTon $(c f .[14, \mathrm{III}])$.

ThÉorème B. - Pour tout $E$, tout $x \geq 1$ et tout entier naturel $k$, on $a$ :

$$
P_{x}\left(\Omega_{E}(n) \geq k\right) \ll_{p_{1}} p_{1}^{-k} \sqrt{1+E(x)} \exp \left(\left(p_{1}-1\right) E(x)\right) ;
$$

pour tout $E$, tout $x \geq 1$ et tout entier naturel $k$ tels que $0 \leq k \leq$ $\log x / \log p_{1}$, on $a$ :

$$
P_{x}\left(\Omega_{E}(n) \geq k\right) \geq \frac{1}{2} p_{1}^{-k} .
$$

K. K. Norton a, d'autre part, annoncé (cf. [14, IV]).

THÉorème C. - Soit $x>3,(\log x)^{-1}<\varepsilon<1$, et supposons que

$$
\begin{gathered}
E(x)>-2\left(p_{1}+2\right) \log \varepsilon+c_{1}\left(p_{2}\right), \\
p_{1} E(x)-\left\{p_{1} E(x)\right\}^{1 / 2} \leq k \leq(1-\varepsilon)(\log x)\left(\log p_{1}\right)^{-1} .
\end{gathered}
$$


Alors

$$
\begin{aligned}
c_{2}\left(p_{2}\right) p_{1}^{-1} \exp \left\{\left(p_{1}-1\right) E(x)\right\} \varepsilon^{p_{1}} \leq \\
P_{x}\left(\Omega_{E}(n) \geq k\right) \leq c_{3}\left(p_{2}\right) p_{1}^{-k} \exp \left\{\left(p_{1}-1\right) E(x)\right\}
\end{aligned}
$$

où chaque $c_{i}\left(p_{2}\right)$ est positive et ne dépend que de $p_{2}$.

Le ThÉorìme $\mathrm{C}$ précise dans une certaine mesure le ThÉorèmE $\mathrm{B}$ en montrant que pour les valeurs de $k \geq p_{1} E(x)$, la loi $P_{x}\left(\Omega_{E}(n) \geq k\right)$ est essentiellement géométrique de raison $1 / p_{1}$. On retrouve ainsi dans le cas général la dichotomie observée dans le cas où $E$ est l'ensemble de tous les nombres premiers.

On voit donc que notre connaissance du comportement local de $\Omega_{E}$ est imparfaite et c'est dans ce contexte que Jean-Louis Nicolas nous en a proposé l'étude. Afin d'énoncer le résultat de nos recherches, nous définissons une certaine classe de lois de probabilité :

Définition. - On appelle loi Poisson-géométrique de paramètre $\lambda \geq 0$ et de raison $r \in] 0,1[$, le produit de convolution $p * g$ de la loi de Poisson de paramètre $\lambda\left(p_{k}=e^{-\lambda} \lambda^{k} / k !, k \in \mathbb{N}\right)$ et de la loi géométrique de raison $r\left(g_{k}=(1-r) r^{k}, k \in \mathbb{N}\right)$. On a :

$$
(p * g)_{k}=(1-r) e^{-\lambda} r^{k} S_{k}\left(\frac{\lambda}{r}\right)
$$

où $S_{k}(X)=1+X+\cdots+X^{k} / k$ ! désigne la $k$-ième somme partielle de la série de $\exp X$.

L'ordre de grandeur des quantités $S_{k}(X)$ est bien connu ( $\left.c f .[15]\right)$. On a en particulier

$$
\begin{aligned}
& S_{k}(X) \asymp \frac{X^{k}}{k !} \quad \text { si } k \leq \alpha X, \quad \alpha \text { fixé dans }[0,1[ \\
& \left.S_{k}(X) \asymp e^{X} \quad \text { si } k \geq \beta X, \quad \beta \text { fixé dans }\right] 1,+\infty[.
\end{aligned}
$$

On a donc :

$$
\begin{aligned}
(p * g)_{k} & \asymp(1-r) e^{-\lambda} \frac{\lambda^{k}}{k !} \quad \text { si } k \leq \alpha \frac{\lambda}{r}, \alpha \text { fixé dans }[0,1[ \\
& \left.\asymp(1-r) e^{\lambda(1 / r-1)} r^{k} \quad \text { si } k \geq \beta \frac{\lambda}{r}, \beta \text { fixé dans }\right] 1,+\infty[.
\end{aligned}
$$

La loi Poisson-géométrique a donc un double comportement : pour $k \leq \alpha \lambda / r$, elle ressemble à une loi de Poisson et pour $k \geq \beta \lambda / r$ elle ressemble à une loi géométrique, la transition ayant lieu dans la

$$
\text { TOME } 117-1989-\mathrm{N}^{\circ} 4
$$


zone $k \sim \lambda / r$. Notre résultat principal est que la loi locale de $\Omega_{E}$ est approximativement Poisson-géométrique.

Soit $x \geq 1, E$ un ensemble de nombres premiers et $k$ un entier naturel. Nous noterons

$$
\begin{array}{ll}
E_{1}=E \backslash\left\{p_{1}\right\}, & E_{1}(u)=\sum_{p \leq u, p \in E_{1}} \frac{1}{p} \\
y=\frac{x}{p_{1}}, & t=\min \left(p_{1}, \frac{k}{E_{1}(y)}\right) .
\end{array}
$$

Nous avons le :

THÉORÈmE. - Il existe des constantes absolues positives $c_{i}$ (avec $4 \leq i \leq 9)$ telles que

$$
P_{x}\left(\Omega_{E}(n)=k\right) \leq c_{4} p_{1}^{-k} \exp \left(c_{5} t-E_{1}(y)\right) S_{k}\left(p_{1} E_{1}(y)\right)
$$

pour tout $x \geq 1$, tout $E$ et tout entier naturel $k$;

(9) $P_{x}\left(\Omega_{E}(n)=k\right) \geq c_{6} p_{1}^{-k} \exp \left(-c_{7} t \log (1+t)-E_{1}(y)\right) S_{k-1}\left(p_{1} E_{1}(y)\right)$

pour tout $x \geq 1$, tout $E$ et tout entier $k \geq 1$ tels que

$$
E_{1}(y) \geq c_{8} t \log (1+t)+c_{9} .
$$

En majorant dans (8) le polynôme $S_{k}$ par la série exponentielle et $t$ par $p_{1}$, on obtient le

Corollaire 1. - Il existe des constantes absolues positives $c_{10}$ et $c_{11}$ telles que

$$
P_{x}\left(\Omega_{E}(n)=k\right) \leq c_{10} p_{1}^{-k} \exp \left(c_{11} p_{1}+\left(p_{1}-1\right) E_{1}(y)\right)
$$

pour tout $x \geq 1$, tout $E$ et tout entier naturel $k$.

A partir de (11), on déduit par sommation une amélioration notable de (5):

$$
P_{x}\left(\Omega_{E}(n) \geq k\right) \leq c_{10}\left(1-p_{1}^{-1}\right)^{-1} p_{1}^{-k} \exp \left(c_{11} p_{1}+\left(p_{1}-1\right) E_{1}(y)\right)
$$

On peut aussi améliorer le THÉORÈme $\mathrm{C}$ :

Corollaire 2. - Soit $x>3,(\log x)^{-1}<\varepsilon<1$, et supposons que

$$
\begin{gathered}
E(x)>-\log \varepsilon+c_{12} p_{1} \log p_{1}, \\
p_{1} E(x)-\left\{p_{1} E(x)\right\}^{1 / 2} \leq k \leq(1-\varepsilon) \log x / \log p_{1} .
\end{gathered}
$$


Alors

$$
\begin{aligned}
& c_{13} p_{1}^{-k} \exp \left\{-c_{14} p_{1} \log p_{1}+\left(p_{1}-1\right) E(x)\right\} \varepsilon^{p_{1}-1} \\
& \leq P_{x}\left(\Omega_{E}(n) \geq k\right) \leq c_{15} p_{1}^{-k} \exp \left(c_{11} p_{1}+\left(p_{1}-1\right) E(x)\right)
\end{aligned}
$$

où chaque $c_{i}$ est une constante positive absolue.

Nous ferons sept remarques concernant notre théorème.

1) Nos raisonnements s'appuient sur la complète additivité de la fonction $\Omega_{E}(n)$ et ne peuvent pas être utilisés pour l'étude de $\omega_{E}(n)=$ $\sum_{p \mid n, p \in E} 1$. On sait en fait que dans le cas où $E$ est l'ensemble de tous les nombres premiers, les comportements asymptotiques des quantités $P_{x}(\Omega(n)=k)$ et $P_{x}(\omega(n)=k)$ sont notablement différents pour $k>2 \log \log x$; le lecteur intéressé pourra consulter [10] et [16].

2) Le fait que la loi de répartition de $\Omega_{E}(n)$ pour $1 \leq n \leq x$ est approximativement Poisson-géométrique admet une interprétation probabiliste simple. Si nous écrivons $\Omega_{E}=v_{p_{1}}+\Omega_{E_{1}}$ où $v_{p_{1}}$ désigne la valuation $p_{1}$-adique, il est facile de voir que la loi de $v_{p_{1}}$ est presque géométrique de raison $1 / p_{1}$ et l'ordre de grandeur de $P_{x}\left(\Omega_{E_{1}}(n)=k\right)$ est, d'après le ThÉonème $\mathrm{A}$, celui d'une loi de Poisson de paramètre $E_{1}(x)$, pour les valeurs "normales" de $k$. Obtenir comme approximation de la loi de $\Omega_{E}$ la convolution de ces lois géométrique et de Poisson traduit l'indépendance approchée des variables aléatoires $v_{p_{1}}(n)$ et $\Omega_{E_{1}}(n)$ pour $1 \leq n \leq x$. Bien entendu, la même analyse permet d'isoler dans la loi de $\Omega_{E_{1}}$ une composante géométrique prépondérante de raison $1 / p_{2}$, mais celle-ci s'avère négligeable par rapport à la loi de $v_{p_{1}}$.

3) Notre théorème a l'intérêt de décrire le comportement de la quantité $P_{x}\left(\Omega_{E}(n)=k\right)$ pour toute valeur de $k$. Néanmoins, dans la zone couverte par le ThÉORÈme A, ce dernier est d'énoncé plus simple et donne dans certains cas un meilleur encadrement que le nôtre.

4) Il serait intéressant de savoir si les quantités $c_{5} t$ et $-c_{7} t \log (1+t)$ qui apparaissent dans (8) et (9) sont ou non optimales. Concernant ces questions nous démontrerons seulement :

on ne peut pas remplacer la quantité $c_{5} t$ de (8) par une quantité $\ll t^{\tau}$, où $\tau=9 / 40+1 / 812-\varepsilon, \varepsilon>0$ quelconque.

5) Toutes les constantes absolues apparaissant dans nos énoncés sont effectivement calculables. Ainsi, en "suivant les constantes" dans le paragraphe 4 à l'aide des majorations explicites de Rosser et SCHOENFELD (cf. [20]) on peut obtenir $c_{10}=200$ et $c_{11}=6$.

TOME $117-1989-\mathrm{N}^{\circ} 4$ 
6) Dans le cas où $E$ est l'ensemble de tous les nombres premiers, l'auteur, H. Delange et J.-L. Nicolas ont précisé notre théorème de la façon suivante ( $c f$. [2] et [1]) : on a uniformément pour $y=x 2^{-k} \rightarrow+\infty$;

$$
P_{x}(\Omega(n)=k) \sim f(t) 2^{-k}(\log y)^{-1} S_{k-1}(2 \log \log y)
$$

où $t=\min (2, k / \log \log y)$ et

$$
f(z)=\frac{1}{2^{z-1} \Gamma(z+1)} \prod\left(1-\frac{1}{p}\right)^{z}\left(1-\frac{z}{p}\right)^{-1},
$$

le produit portant sur les nombres premiers impairs.

Historiquement, le premier résultat dans cette direction est dû à HARDY et Ramanujan ( $c f$. [17, p. 271]) : on a uniformément pour $x \geq 3$,

$$
P_{x}(\Omega(n)=k) \leq C_{0}\left(\frac{10}{9}\right)^{-k}(\log x)^{-1} S_{k-1}\left(\frac{10}{9}\left(\log \log x+C_{1}\right)\right),
$$

où $C_{0}$ et $C_{1}$ sont des constantes positives absolues.

7) On peut se demander si une formule asymptotique valable pour tout $k$ existe pour $P_{x}\left(\Omega_{E}(n)=k\right)$. Les méthodes développées par $\mathrm{G}$. HaLÁsz ( $c f$. [6]) permettent peut-être d'aborder ce problème.

Le paragraphe 2 expose la structure de la démonstration du théorème. La paragraphe 3 donne quelques lemmes utiles dans la suite, le théorème étant démontré aux paragraphes 4,5 et 6 . Enfin, le Corollaire 2 et l'assertion (14) sont l'objet des paragraphes 7 et 8 .

Nous tenons à remercier J.-L. Nicolas et G. Tenenbaum pour leurs conseils et encouragements, sans lesquels les résultats qui précèdent eussent été moins précis et les démonstrations qui suivent plus compliquées. G. HALÁsz et K.K. NoRTON nous ont également fait d'utiles remarques sur une première version de cet article.

\section{Structure de la démonstration}

L'idée de départ, comme dans [12], est dûe à G. HaLÁsz. Ecrivons $n=p_{1}^{\alpha} n^{\prime}$, où $\left(n^{\prime}, p_{1}\right)=1$. On a alors :

$$
\begin{aligned}
\left(n \leq x \text { et } \Omega_{E}(n)=k\right) & \Longleftrightarrow\left(\alpha \leq k, \Omega_{E}\left(n^{\prime}\right)=k-\alpha \text { et } p_{1}^{\alpha} n^{\prime} \leq x\right) \\
& \Longleftrightarrow\left(\Omega_{E}\left(n^{\prime}\right) \leq k \text { et } n^{\prime} p_{1}^{-\Omega_{E}\left(n^{\prime}\right)} \leq y\right),
\end{aligned}
$$

BULLETIN DE LA SOCIÉTÉ MATHÉMATIQUE DE FRANCE 
où $y=x p_{1}^{-k}$. Ainsi,

$$
\sum_{n \leq x, \Omega_{E}(n)=k} 1=\sum_{\psi(n) \leq y, \Omega_{E}(n) \leq k}^{*} 1
$$

où l'étoile indique une sommation ne portant que sur les entiers premiers à $p_{1}$, et où $\psi(n)=n p_{1}^{-\Omega_{E}(n)} ; \psi$ est une fonction complètement multiplicative, tendant vers l'infini avec $n,\left(n, p_{1}\right)=1$.

Nous commencerons par démontrer le Corollaire 1, en supprimant la condition $\Omega_{E}(n) \leq k$ de la dernière somme :

$$
C(y)=\sum_{\psi(n) \leq y}^{*} 1 \leq c_{10} e^{c_{11} p_{1}} y \exp \left(\left(p_{1}-1\right) E_{1}(y)\right), \quad y \geq 1
$$

Pour démontrer la majoration (8) du théorème, il nous suffira donc, d'après le Lemme 6, d'obtenir :

$$
C(y, \ell)=\sum_{\substack{\psi(n) \leq y \\ \Omega_{E}(n)=\ell}}^{*} 1 \ll e^{c_{5} t-E_{1}(y)} y \frac{\left(p_{1} E_{1}(y)\right)^{\ell}}{\ell !}
$$

uniformément pour $y \geq 1,0 \leq \ell<p_{1} E_{1}(y)$, où $t=\ell / E_{1}(y)$.

Pour démontrer la minoration (9) du théorème, le LEMME 6 nous montre encore qu'il suffit d'avoir :

$$
C(y, \ell)+C(y, \ell-1) \gg e^{-c_{7} t \log (1+t)-E_{1}(y)} y \frac{\left(p_{1} E_{1}(y)\right)^{\ell-1}}{(\ell-1) !},
$$

uniformément pour $y \geq 1,1 \leq \ell<p_{1} E_{1}(y), E_{1}(y) \geq c_{8} t \log (1+t)+c_{9}$, où $t=\ell / E_{1}(y)$.

Pour (19), nous utilisons une autre idée de G. HalÁsz ( $c f$. [7]), qui a remarqué qu'on peut beaucoup plus facilement minorer

$$
P_{x}\left(\Omega_{E}(n)=k\right)+P_{x}\left(\Omega_{E}(n)=k-1\right)
$$

que $P_{x}\left(\Omega_{E}(n)=k\right)$. Nous évitons ainsi le recours à l'argument élémentaire très astucieux de [18].

Pour démontrer (17), (18) et (19) nous utiliserons la méthode élémentaire, essentiellement dûe à Tchebycheff, qui consiste à estimer successivement les sommes $\sum^{*} 1 / \psi(n), \sum^{*} \log \psi(n)$ et $\sum^{*} 1$ correspondantes (pour une application de cette méthode à un autre problème, $c f$. [13]). Pour les

TOME $117-1989-\mathrm{N}^{\circ} 4$ 
majorations, nous adopterons la forme très simple contenue dans l'exercice 00 de [8].

Dans les trois cas, l'étape cruciale est l'estimation de la somme $\sum^{*} 1 / \psi(n)$. Pour (17), elle se fait par comparaison avec un produit eulérien fini et, pour (18) et (19), par comparaison avec un développement multinomial et par la méthode de Rankin. Observons qu'à l'étape correspondante de [7], G. HALÁsz utilisait une méthode analytique, alors que les démonstrations qui suivent sont entièrement élémentaires. Notons enfin que (18) est obtenue en deux temps, une inégalité moins bonne étant démontrée d'abord, puis réutilisée pour donner (18). La quantité $c_{5} t$ de (8) et (18) remplace avantageusement la quantité $c p_{1} \log \log \left(1+p_{1}\right)$ qui apparaît dans une première version de notre article $(c f$. [1]). G. TenenBAUM nous a indiqué la méthode élémentaire du paragraphe 5 , qui permet de supprimer le facteur $\log \log \left(1+p_{1}\right)$ et d'obtenir finalement $c_{5} t$.

\section{Résultats auxiliaires}

LeMme 1. - On a uniformément :

$$
\prod_{p \leq y, p \in E_{1}}\left(1-\frac{p_{1}}{p}\right)^{-1} \leq \exp \left\{\mathrm{O}\left(p_{1}\right)+p_{1} E_{1}(y)\right\} .
$$

Démonstration. - On peut supposer $y \geq p_{2}$, et il s'agit de démontrer que

$$
\sum_{p \leq y, p \in E_{1}} \varphi\left(\frac{p_{1}}{p}\right) \ll p_{1}, \quad \text { où } \varphi(t)=\log \frac{1}{1-t}-t=\sum_{k \geq 2} \frac{t^{k}}{k} .
$$

Or on a :

$$
\begin{aligned}
\sum_{p \leq y, p \in E_{1}} \varphi\left(\frac{p_{1}}{p}\right) & \leq \sum_{p \geq p_{2}} \varphi\left(\frac{p_{1}}{p}\right) \\
& =\int_{p_{2}^{-}}^{+\infty} \varphi\left(\frac{p_{1}}{t}\right) d \pi(t) \\
& \ll \frac{1}{\log p_{2}} \int_{p_{2}}^{+\infty} \frac{p_{1}}{t} \varphi^{\prime}\left(\frac{p_{1}}{t}\right) d t \\
& =\frac{p_{1}}{\log p_{2}} \int_{0}^{p_{1} / p_{2}} \varphi^{\prime}(u) \frac{d u}{u} \\
& =\frac{p_{1}}{\log p_{2}} \log \left(\frac{p_{2}}{p_{2}-p_{1}}\right) \ll p_{1} .
\end{aligned}
$$

BULLETIN DE LA SOCIÉTÉ MATHÉMATIQUE DE FRANCE 
On a utilisé ici l'intégration par parties, l'inégalité $\pi(t) \ll t / \log t$ et l'égalité $\varphi^{\prime}(u)=u / 1-u$.

LEMme 2. - On a uniformément pour $y \geq 1$ et $\beta \geq 0$ :

$$
\begin{aligned}
E_{1}\left(p_{1}^{\beta} y\right)-E_{1}(y) & \leq \log (1+\beta)+\mathrm{O}(1) \\
E_{1}\left(y^{1+\beta}\right)-E_{1}(y) & \leq \log (1+\beta)+\mathrm{O}(1) .
\end{aligned}
$$

Démonstration. - Si l'on pose $y^{*}=\max \left(y, p_{2}\right)$, on a :

$$
\begin{aligned}
E_{1}\left(p_{1}^{\beta} y\right)-E_{1}(y) & \leq \sum_{y^{*} \leq p \leq p_{1}^{\beta} y^{*}} \frac{1}{p} \\
& \leq \log \frac{\log \left(p_{1}^{\beta} y^{*}\right)}{\log \left(y^{*}\right)}+\mathrm{O}(1) \leq \log (1+\beta)+\mathrm{O}(1) \\
E_{1}\left(y^{1+\beta}\right)-E_{1}(y) & \leq \sum_{y^{*} \leq p \leq\left(y^{*}\right)^{1+\beta}} \frac{1}{p} \\
& \leq \log \frac{\log \left(\left(y^{*}\right)^{1+\beta}\right)}{\log \left(y^{*}\right)}+\mathrm{O}(1)=\log (1+\beta)+\mathrm{O}(1)
\end{aligned}
$$

LEMME 3. - On a uniformément pour $y \geq 1$ :

$$
\sum_{p^{\nu} \leq y, p \notin E} \log \left(p^{\nu}\right)+\sum_{\left(p / p_{1}\right)^{\nu} \leq y, p \in E_{1}} \log \left(\left(\frac{p}{p_{1}}\right)^{\nu}\right) \ll p_{1}^{2} y .
$$

De plus, on $a$ :

$$
\sum_{p \leq y, p \notin E} \log p+\sum_{p \leq p_{1} y, p \in E_{1}} \log \frac{p}{p_{1}} \geq \frac{1}{2} y \quad \text { pour } y \geq p_{1}^{8} .
$$

Démonstration. - Pour (22), nous majorons chaque terme des deux sommes par $\log y$ et nous démontrons que :

$$
\sum_{p^{\nu} \leq y, p \notin E} 1+\sum_{\nu \geq 1} D_{\nu}(y) \ll \frac{p_{1}^{2} y}{\log y}
$$

où $D_{\nu}(y)=\sum_{p \leq p_{1} y^{1 / \nu}, p \in E_{1}} 1$. Les sommes $\sum_{p^{\nu} \leq y, p \notin E} 1$ et $D_{1}(y)$ étant respectivement de l'ordre de $y / \log y$ et de $p_{1} y / \log y$, il nous suffit de montrer que :

$$
\sum_{\nu \geq 2} \sum_{p_{2} \leq p \leq p_{1} y^{1 / \nu}} 1 \ll p_{1}^{2} \frac{y}{\log y} .
$$

TOME $117-1989-\mathrm{N}^{\circ} 4$ 
En effet, la somme sur $p$ est nulle dès que $\nu>(\log y) /\left(\log \left(p_{2} / p_{1}\right)\right)$ et elle est de toutes façons $\leq p_{1} \sqrt{y}$. La somme double considérée est donc :

$$
\begin{aligned}
& \leq \frac{p_{1}}{\log \left(p_{2} / p_{1}\right)} \sqrt{y} \log y \\
& \ll p_{1}^{2} \frac{y}{\log (y)} \quad \text { car } p_{2} \geq p_{1}+1 .
\end{aligned}
$$

Pour (23), nous avons :

$$
\begin{aligned}
\sum_{p \leq y, p \notin E} \log p+\sum_{p \leq p_{1} y, p \in E_{1}} \log \frac{p}{p_{1}} & \geq \sum_{p \leq y} \log \left(\frac{p}{p_{1}}\right) \\
& =\sum_{p \leq y} \log p-\log p_{1} \sum_{p \leq y} 1 \\
& \geq \frac{3}{4} y-2 \log p_{1} \frac{y}{\log y} \\
& \geq \frac{1}{2} y \text { pour } y \geq p_{1}^{8} .
\end{aligned}
$$

Lemme 4. - Pour $y>1$ et $\sigma=1+1 / \log y$, on a

$$
\sum_{p \in E_{1}} p^{-\sigma}=E_{1}(y)+\mathrm{O}(1) .
$$

Démonstration. - Ce résultat est classique. On a :

$$
\begin{aligned}
\left|\sum_{p \in E_{1}} p^{-\sigma}-E_{1}(y)\right| & \leq(\sigma-1) \sum_{p \leq y} \frac{\log p}{p}+\sum_{p>y} p^{-\sigma} \\
& \ll 1+\frac{\sigma}{\log y} \int_{y}^{+\infty} t^{-\sigma} d t \ll 1
\end{aligned}
$$

si $y \geq 2$, ce que l'on peut supposer.

Lemme 5. - On a uniformément pour $0 \leq v \leq p_{1}+0,9$ :

$$
\prod_{p \in E_{1}}\left(1-v^{2} p^{-2}\right)^{-1} \ll e^{\mathrm{O}(v)} .
$$

Démonstration. - Il suffit de vérifier que :

$$
\int_{p_{2}^{-}}^{+\infty} \log \left(\frac{1}{1-v^{2} t^{-2}}\right) d \pi(t) \ll v .
$$

BULLETIN DE LA SOCIÉTÉ MATHÉMATIQUE DE FRANCE 
En intégrant par parties, le premier membre est :

$$
\begin{aligned}
& \ll v^{2} \int_{p_{2}}^{+\infty} \frac{d t}{\left(t^{2}-v^{2}\right) \log t} \\
& \ll \frac{v}{\log p_{2}} \log \frac{p_{2}-v}{p_{2}-v} \\
& \ll v .
\end{aligned}
$$

Lemme 6. - Pour $x$ réel $\geq 0$ et $k$ entier $\geq 0$, on $a:$

$$
\begin{aligned}
& S_{k}(x) \gg e^{x} \quad \text { si } k \geq x-\sqrt{x}-1, \\
& S_{k}(x) \ll \sqrt{k} \frac{x^{k}}{k !} \quad \text { si } k<x .
\end{aligned}
$$

Démonstration. - (24) est très classique. Pour (25) on remarque que $S_{k}(x) \leq e^{x} \ll \sqrt{k} x^{k} / k$ ! si $x-\sqrt{x} \leq k<x$, d'après la formule de Stirling; et $S_{k}(x)=x^{k} / k !+x^{k-1} /(k-1) !+\cdots \leq\left(x^{k} / k !\right)(1 /(1-k / x)) \ll \sqrt{k} x^{k} / k !$ si $k<x-\sqrt{x}$. Pour des estimations beaucoup plus précises, le lecteur peut consulter [15].

\section{Démonstration de (17)}

Pour $y \geq 1$, posons :

$$
A(y)=\sum_{\psi(n) \leq y}^{*} \frac{1}{\psi(n)} \text { et } B(y)=\sum_{\psi(n) \leq y}^{*} \log \psi(n) .
$$

En utilisant la formule de Mertens, le Lemme 1 et le Lemme 2, nous avons :

$$
\begin{aligned}
A(y) & \leq \prod_{p \leq y, p \notin E}\left(1-\frac{1}{p}\right)^{-1} \prod_{p \leq p_{1} y, p \in E_{1}}\left(1-\frac{p_{1}}{p}\right)^{-1} \\
& \ll \log (2 y) \exp \left\{\mathrm{O}\left(p_{1}\right)+p_{1} E_{1}\left(p_{1} y\right)-E_{1}(y)\right\} \\
& \ll \log (2 y) \exp \left\{\mathrm{O}\left(p_{1}\right)+\left(p_{1}-1\right) E_{1}(y)\right\} .
\end{aligned}
$$

On écrit maintenant :

$$
\begin{aligned}
B(y) & =\sum_{\psi(n) \leq y}^{*} \log \psi(n)=\sum_{\substack{\psi\left(m p^{\nu}\right) \leq y \\
(m, p)=1}}^{*} \log \psi\left(p^{\nu}\right) \\
& \leq \sum_{\psi(m) \leq y}\left\{\sum_{\substack{p^{\nu} \leq y / \psi(m) \\
p \notin E}} \log \left(p^{\nu}\right)+\sum_{\substack{\left(p / p_{1}\right)^{\nu} \leq y / \psi(m) \\
p \in E_{1}}} \log \left(\left(\frac{p}{p_{1}}\right)^{\nu}\right)\right\}
\end{aligned}
$$$$
\text { томе } 117-1989-\mathrm{N}^{\circ} 4
$$ 
D'après le LEMmE 3 , cette dernière quantité est :

$$
\begin{aligned}
& \ll p_{1}^{2} y A(y) \\
& \ll y \log (2 y) \exp \left\{\mathrm{O}\left(p_{1}\right)+\left(p_{1}-1\right) E_{1}(y)\right\}
\end{aligned}
$$

Enfin,

$$
\begin{aligned}
\log (2 y) C(y) & =B(y)+\sum_{\psi(n) \leq y}^{*} \log \frac{2 y}{\psi(n)} \\
& \leq B(y)+2 y A(y) \\
& \ll y \log (2 y) \exp \left\{\mathrm{O}\left(p_{1}\right)+\left(p_{1}-1\right) E_{1}(y)\right\} \text { d'où (17). }
\end{aligned}
$$

\section{Démontration de (18)}

Nous posons naturellement :

$$
A(y, \ell)=\sum_{\substack{\psi(n) \leq y \\ \Omega_{E}(n)=\ell}}^{*} \frac{1}{\psi(n)} \quad \text { et } \quad B(y, \ell)=\sum_{\substack{\psi(n) \leq y \\ \Omega_{E}(n)=\ell}}^{*} \log \psi(n) .
$$

Désignons par $a$ (resp. $b$ ) un entier générique qui vérifie la condition $\Omega_{E}(a)=0$ (resp. $p \mid b \Rightarrow p \in E_{1}$ ). Pour majorer $A(y, \ell)$ nous allons utiliser la décomposition $n=a b$ et la méthode de Rankin :

$$
A(y, \ell)=p_{1}^{\ell} \sum_{a b \leq p_{1}^{\ell} y, \Omega(b)=\ell} \frac{1}{a b} \leq p_{1}^{\ell} \sum_{\Omega(b)=\ell}\left(\frac{p_{1}^{\ell} y}{b}\right)^{\sigma} \frac{1}{b} \sum_{a \leq y} \frac{1}{a}
$$

où $\sigma>0$, en utilisant le fait que $b>p_{1}^{\ell}$. Dans la suite, nous choisissons $\sigma=1 / \log y\left(\right.$ on a $\left.y \geq p_{2} \operatorname{car} E_{1}(y)>0\right)$. On a :

$$
\sum_{a \leq y} \frac{1}{a} \leq \prod_{p \leq y, p \notin E}\left(1-\frac{1}{p}\right)^{-1} \ll \log y e^{-E_{1}(y)} .
$$

De plus,

$$
p_{1}^{\ell \sigma}=\exp \left\{\frac{\ell}{E_{1}(y)} \frac{E_{1}(y) \log p_{1}}{\log y}\right\} \leq \exp (\mathrm{O}(t))
$$

car

$$
E_{1}(y) \leq \sum_{p_{1}<p \leq y} \frac{1}{p} \leq \log \left(\frac{\log y}{\log p_{1}}\right)+\mathrm{O}(1) \ll \frac{\log y}{\log p_{1}}
$$


On peut donc écrire :

$$
A(y, \ell) \ll \log y \cdot p_{1}^{\ell} e^{\mathrm{O}(t)-E_{1}(y)} \sum_{\Omega(b)=\ell} \frac{1}{b^{1+\sigma}} .
$$

Le lemme suivant sera également utilisé au paragraphe 6.

Lemme 7. - Pour $q>0$, on pose $f(\sigma)=\sum_{\Omega(b)=\ell} b^{-1-\sigma}$, où b désigne un entier générique qui vérifie la condition $p \mid b \Rightarrow p \in E_{1}$. Si l'on a $0 \leq \ell<\left(p_{1}+0,4\right) E_{1}(y)$ et $\sigma=1 / \log y$, alors

$$
f(\sigma) \ll e^{\mathrm{O}(t)} \frac{E_{1}(y)^{\ell}}{\ell !}, \quad \text { ò̀ } t=\frac{\ell}{E_{1}(y)} .
$$

Démonstration. - On décompose chaque $b$ sous la forme $b=r s^{2}$ avec $\mu(r)^{2}=1$ d'où

$$
\sum_{\Omega(b)=\ell} \frac{1}{b^{1+\sigma}}=\sum_{j+2 h=\ell} \sum_{\omega(r)=j} \frac{\mu(r)^{2}}{r^{1+\sigma}} \sum_{\Omega(s)=h} \frac{1}{s^{2+2 \sigma}} .
$$

D'une part

$$
\sum_{\omega(r)=j} \frac{\mu(r)^{2}}{r^{1+\sigma}} \leq \frac{1}{j !}\left(\sum_{p \in E_{1}} \frac{1}{p^{1+\sigma}}\right)^{j}
$$

ce qui par le Lemme 4 est

$$
\begin{aligned}
& \leq \frac{1}{j !}\left(E_{1}(y)+\mathrm{O}(1)\right)^{j} \\
& \leq e^{\mathrm{O}(t)} \frac{E_{1}(y)^{\ell}}{\ell !}
\end{aligned}
$$

D'autre part, pour tout $v \geq 1$,

$$
\begin{aligned}
\sum_{\Omega(s)=h} \frac{1}{s^{2}} & \leq v^{-h} \sum \frac{v^{\Omega(s)}}{s^{2}} \\
& =v^{-h} \prod_{p \in E_{1}}\left(1-\frac{v}{p^{2}}\right)^{-1}
\end{aligned}
$$

TOME $117-1989-\mathrm{N}^{\circ} 4$ 
Si on choisit $v=\left(t+\frac{1}{2}\right)^{2}$, le produit ci-dessus est $\ll e^{\mathrm{O}(t)}$ d'après le Lemme 5. Par conséquent :

$$
\begin{aligned}
\sum_{\Omega(b)=\ell} \frac{1}{b^{1+\sigma}} & \ll e^{\mathrm{O}(t)} \sum_{j+2 h=\ell} \frac{E_{1}(y)^{j}}{j !}\left(t+\frac{1}{2}\right)^{-2 h} \\
& \ll e^{\mathrm{O}(t)} \frac{E_{1}(y)^{\ell}}{\ell !} \sum_{h=0}^{+\infty}\left(\frac{\ell}{E_{1}(y)\left(t+\frac{1}{2}\right)}\right)^{2 h} \\
& \ll e^{\mathrm{O}(t)} \frac{E_{1}(y)^{\ell}}{\ell !}
\end{aligned}
$$

ce qui démontre le Lemme 7 . On a donc bien :

$$
A(y, \ell) \ll \log y \cdot e^{\mathrm{O}(t)-E_{1}(y)} \frac{\left(p_{1} E_{1}(y)\right)^{\ell}}{\ell !} .
$$

Nous majorons maintenant $B(y, \ell)$ :

$$
\begin{gathered}
B(y, \ell)=\sum_{\substack{\psi\left(m p^{\nu}\right) \leq y,(m, p)=1 \\
\Omega_{E}(m)+\nu \Omega_{E}(p)=\ell}} \log \psi\left(p^{\nu}\right) \\
\leq \sum_{\nu=1}^{\ell} \sum_{\substack{\psi(m) \leq y \\
\Omega_{E}(m)=\ell-\nu}}^{*} \sum_{\substack{p \leq p_{1}(y / \psi(m))^{1 / \nu} \\
p \in E_{1}}} \log \left(\left(\frac{p}{p_{1}}\right)^{\nu}\right) \\
+\sum_{\substack{\psi(m) \leq y \\
\Omega_{E}(m)=\ell}}^{*} \sum_{\substack{\nu \leq y / \psi(m) \\
p \notin E}} \log \left(p^{\nu}\right) .
\end{gathered}
$$

En observant que

$$
\begin{aligned}
\sum_{\substack{p \leq p_{1}(y / \psi(m))^{1 / \nu} \\
p \in E_{1}}} \log \left(\frac{p}{p_{1}}\right)^{\nu} & \leq \log \left(\frac{y}{\psi(m)}\right) \pi\left(\frac{p_{1} y}{\psi(m)}\right) \\
& \ll \frac{p_{1} y}{\psi(m)}
\end{aligned}
$$

nous obtenons :

$$
\begin{aligned}
B(y, \ell) & \ll y A(y, \ell)+\sum_{\nu=1}^{\ell} p_{1} y A(y, \ell-\nu) \\
& \ll y \log y \cdot e^{\mathrm{O}(t)-E_{1}(y)} \frac{\left(p_{1} E_{1}(y)^{\ell}\right.}{\ell !} \\
& \quad+\sum_{\nu=1}^{\ell} y(\log y) p_{1} e^{\mathrm{O}(t)-E_{1}(y)} \frac{\left(p_{1} E_{1}(y)\right)^{\ell-\nu}}{(\ell-\nu) !} \\
& \ll y \log y e^{\mathrm{O}(t)-E_{1}(y)} S_{\ell}\left(p_{1} E_{1}(y)\right)
\end{aligned}
$$

BULLETIN DE LA SOCIÉTÉ MATHÉMATIQUE DE FRANCE 
car, pour $\nu=1, \ldots, \ell$,

$$
p_{1} \frac{\left(p_{1} E_{1}(y)\right)^{\ell-\nu}}{(\ell-\nu) !} \leq t \frac{\left(p_{1} E_{1}(y)\right)^{\ell-\nu+1}}{(\ell-\nu+1) !}
$$

On en déduit, d'après le Lemme 6 :

$$
B(y, \ell) \ll y(\log y) e^{\mathrm{O}(t)-E_{1}(y)} \sqrt{\ell} \frac{\left(p_{1} E_{1}(y)\right)^{\ell}}{\ell !}
$$

puis, comme au paragraphe 4 ,

$$
C(y, \ell) \ll y e^{\mathrm{O}(t)-E_{1}(y)} \sqrt{\ell} \frac{\left(p_{1} E_{1}(y)\right)^{\ell}}{\ell !} .
$$

Pour supprimer le facteur superflu $\sqrt{\ell}$, on réutilise (27) sous la forme :

$$
B(y, \ell) \ll y\left(A(y, \ell)+p_{1} A(y, \ell-1)\right)+p_{1} \sum_{\nu=2}^{\ell} \nu \sum_{\substack{\psi(m) \leq y \\ \Omega_{E}(m)=\ell-\nu}}\left(\frac{y}{\psi(m)}\right)^{1 / v} .
$$

D'après l'inégalité de Hölder, la somme intérieure est

$$
\begin{aligned}
& \ll(y A(y, \ell-\nu))^{1 / \nu}(C(y, \ell-\nu))^{((\nu-1) / \nu)} \\
& \ll y e^{\mathrm{O}(t)-E_{1}(y)} \frac{\left(p_{1} E_{1}(y)\right)^{\ell-\nu}}{(\ell-\nu) !}(\log y)^{1 / \nu} \ell^{((\nu-1) /(2 \nu))} .
\end{aligned}
$$

La somme en $\nu$ est donc :

car

$$
\begin{aligned}
& \ll y e^{\mathrm{O}(t)-E_{1}(y)} \sum_{\nu=2}^{\ell} \nu \ell^{((\nu-1) /(2 \nu))}(\log y)^{1 / \nu} \frac{\left(p_{1} E_{1}(y)\right)^{\ell-\nu}}{(\ell-\nu) !} \\
& \ll y e^{\mathrm{O}(t)-E_{1}(y)} \ell^{5 / 2} \sqrt{\log y} \frac{\left(p_{1} E_{1}(y)\right)^{\ell-2}}{(\ell-2) !} \\
& \ll y \log y \cdot e^{\mathrm{O}(t)-E_{1}(y)} \frac{\left(p_{1} E_{1}(y)\right)^{\ell-2}}{(\ell-2)}
\end{aligned}
$$

$$
\ell^{5 / 2}=t^{5 / 2} E_{1}(y)^{5 / 2} \ll t^{5 / 2} \sqrt{\log y} .
$$

Finalement, on a bien :

$$
\begin{aligned}
B(y, \ell) & \ll y \log y \cdot e^{\mathrm{O}(t)-E_{1}(y)}\left\{\frac{\left(p_{1} E_{1}(y)\right)^{\ell}}{\ell !}+p_{1} \frac{\left(p_{1} E_{1}(y)\right)^{\ell-1}}{(\ell-1) !}\right\} \\
& \ll y \log y \cdot e^{\mathrm{O}(t)-E_{1}(y)} \frac{\left(p_{1} E_{1}(y)\right)^{\ell}}{\ell !}, \\
C(y, \ell) & \ll y e^{\mathrm{O}(t)-E_{1}(y)} \frac{\left(p_{1} E_{1}(y)\right)^{\ell}}{\ell !} .
\end{aligned}
$$




\section{Démonstration de (19)}

Afin de démontrer (19), nous allons énoncer cinq nouveaux lemmes. Dans ce paragraphe, nous reprenons la notation $f(\sigma)=\sum_{\Omega(b)=\ell} b^{-1-\sigma}$ pour $\sigma>0$, où $b$ désigne un entier générique tel que $p \mid b \Rightarrow p \in E_{1}$. Les $a_{i}$ sont des constantes positives absolues.

LEMME 8. - Si $E_{1}(y) \geq a_{0}$ et $\sigma=1 / \log y$, on $a$ :

$$
f(\sigma) \geq e^{-a_{1} t} \frac{E_{1}(y)^{\ell}}{\ell !}, \quad \text { où } \quad t=\frac{\ell}{E_{1}(y)} .
$$

Démonstration. - On a :

$$
\begin{aligned}
f(\sigma) & \geq \frac{1}{\ell !}\left(\sum_{p \in E_{1}} p^{-1-\sigma}\right)^{\ell} \quad \text { ce qui, d'après le Lemme } 4, \text { est : } \\
& \geq \frac{1}{\ell !}\left(E_{1}(y)+O(1)\right)^{\ell} \\
& \geq e^{-a_{1} t} \frac{E_{1}(y)^{\ell}}{\ell !} \quad \text { si } E_{1}(y) \geq a_{0}, \text { d'après le Lemme } 4 .
\end{aligned}
$$

Lemme 9. - Il existe une constante absolue $K>0$ telle que, si $0 \leq \ell<\left(p_{1}+0,3\right) E_{1}(y)$ et $E_{1}(y) \geq a_{2} t \log (1+t)+a_{3}$, alors

$$
f(\sigma)-y^{-\sigma^{\prime}} f\left(\sigma-\sigma^{\prime}\right) \gg e^{-a_{4} t \log (1+t)} \frac{E_{1}(y)^{\ell}}{\ell !},
$$

où $\sigma=2 K(t+1) / \log y, \sigma^{\prime}=K(t+1) / \log y$ et $t=\ell / E_{1}(y)$.

Démonstration. - Posons $y_{1}=y^{1 / 2 K(t+1)}, y_{2}=y^{1 / K(t+1)}=y_{1}^{2}$, $t_{1}=\ell / E_{1}\left(y_{1}\right)$ et $t_{2}=\ell / E_{1}\left(y_{2}\right)$. En supposant que $K$ et $y \geq 1$, nous avons :

$$
y_{1} \leq y_{2} \leq y \quad \text { d'où } \quad E_{1}\left(y_{1}\right) \leq E_{1}\left(y_{2}\right) \leq E_{1}(y) \quad \text { et } \quad t_{1} \geq t_{2} \geq t .
$$

D'autre part, le Lemme 2 nous donne :

$$
\begin{aligned}
& E_{1}\left(y_{1}\right) \geq E_{1}(y)-\log (t+1)-\log (2 K)-a_{5} \\
& E_{1}\left(y_{2}\right) \leq E_{1}\left(y_{1}\right)+a_{6}
\end{aligned}
$$

Par conséquent, si

$$
E_{1}(y) \geq \log (t+1)+\log (2 K)+a_{5}+a_{0},
$$

BULLETIN DE LA SOCIÉTÉ MATHÉMATIQUE DE FRANCE 
le Lemme 8 nous donne

$$
f(\sigma) \geq e^{-a_{1} t_{1}} \frac{E_{1}\left(y_{1}\right)^{\ell}}{\ell !} .
$$

Si maintenant

$$
E_{1}(y) \geq 2\left(\log (t+1)+\log (2 K)+a_{5}\right)
$$

nous aurons, en utilisant l'inégalité $1-u \geq \exp (-u \log 4)$ pour $0 \leq u \leq \frac{1}{2}$ :

$$
E_{1}\left(y_{1}\right)^{\ell} \geq \exp \left(-t \log 4\left(\log (t+1)+\log (2 K)+a_{5}\right)\right) E_{1}(y)^{\ell}
$$

et si, en outre,

$$
E_{1}(y) \geq 20 t\left(\log (t+1)+\log (2 K)+a_{5}\right)
$$

alors

$$
\begin{aligned}
t_{2} & \leq t_{1}=\frac{\ell}{E_{1}\left(y_{1}\right)} \\
& \leq \frac{\ell}{\left(E_{1}(y)-\log (t+1)-\log (2 K)-a_{5}\right)} \\
& \leq \frac{\ell}{E_{1}(y)}\left(1+2 \frac{\log (t+1)+\log (2 K)+a_{5}}{E_{1}(y)}\right) \\
& \leq t+0,1 .
\end{aligned}
$$

Supposons maintenant (29), (31) et (33) simultanément vérifiées. Nous pouvons réécrire (30) sous la forme

$$
f(\sigma) \geq a_{7} \exp \left(-a_{1} t\right) \frac{E_{1}\left(y_{1}\right)^{\ell}}{\ell !} .
$$

De plus, si $0 \leq \ell<\left(p_{1}+0,3\right) E_{1}(y)$, on a $0 \leq \ell<\left(p_{1}+0,4\right) E_{1}\left(y_{2}\right)$. Donc, d'après le Lemme 7 ,

$$
\begin{aligned}
y^{-\sigma^{\prime}} f\left(\sigma-\sigma^{\prime}\right) & \leq a_{8} \exp \left(-K(t+1)+a_{9} t_{2}\right) \frac{E_{1}\left(y_{2}\right)^{\ell}}{\ell !} \\
& \leq a_{10} \exp \left(-K(t+1)+a_{11} t\right) \frac{E_{1}\left(y_{1}\right)^{\ell}}{\ell !}
\end{aligned}
$$

Choisissons $K$ tel que $K \geq a_{1}+a_{11}$ et $a_{10} e^{-K} \leq a_{7} / 2$; (34) et (35) prouvent que

$$
f(\sigma)-y^{-\sigma^{\prime}} f\left(\sigma-\sigma^{\prime}\right) \geq \frac{1}{2} a_{7} \exp \left(-a_{1} t\right) \frac{E_{1}\left(y_{1}\right)^{\ell}}{\ell !} .
$$

TOME $117-1989-\mathrm{N}^{\circ} 4$ 
Compte tenu de (32), nous obtenons :

$$
\begin{aligned}
f(\sigma)-y^{-\sigma^{\prime}} f\left(\sigma-\sigma^{\prime}\right) & \gg \exp \left(-a_{12} t \log (1+t)-a_{13} t\right) \frac{E_{1}(y)^{\ell}}{\ell !} \\
& \gg e^{-a_{4} t \log (1+t)} \frac{E_{1}(y)^{\ell}}{\ell !} .
\end{aligned}
$$

Pour terminer, on observe que si $a_{2}$ et $a_{3}$ sont assez grands, la condition

$$
E_{1}(y) \geq a_{2} t \log (1+t)+a_{3}
$$

entraîne (29), (31) et (33).

LEMME 10. - On a :

$$
\sum_{\substack{b \leq \sqrt{y} p_{1}^{-8} \\ \Omega(b)=\ell}} \frac{1}{b} \gg \exp \left(-a_{14} t \log (1+t)\right) \frac{E_{1}(y)^{\ell}}{\ell !}
$$

pourvu que $E_{1}(y) \geq a_{15} t \log (1+t)+a_{16}$ et $0 \leq \ell<p_{1} E_{1}(y)$, où $t=\ell / E_{1}(y)$.

Démonstration. - D'après (20) et (21), on a :

$$
E_{1}\left(y^{1 / 2} p_{1}^{-8}\right) \geq E_{1}(y)-a_{17} .
$$

$\mathrm{Si}$, en supposant $E_{1}\left(y^{1 / 2} p_{1}^{-8}\right)>0$, nous posons $y_{3}=y^{1 / 2} p_{1}^{-8}$ ainsi que $t_{3}=\ell / E_{1}\left(y_{3}\right)$, nous aurons

$$
t_{3} \leq \frac{\ell}{E_{1}(y)-a_{17}} \leq \frac{\ell}{E_{1}(y)}\left(1+2 \frac{a_{17}}{E_{1}(y)}\right) \leq t+0,3
$$

si

$$
E_{1}(y) \geq \frac{20}{3} a_{17} t+2 a_{17}
$$

Par conséquent, en choisissant $a_{15}$ et $a_{16}$ assez grands, les inégalités

$$
E_{1}(y) \geq a_{15} t \log (1+t)+a_{16} \text { et } 0 \leq \ell<p_{1} E_{1}(y)
$$

entraînent (36) et les inégalités

$$
E_{1}\left(y_{3}\right) \geq a_{2} t_{3} \log \left(1+t_{3}\right)+a_{3} \quad \text { et } \quad 0 \leq \ell<\left(p_{1}+0,3\right) E_{1}\left(y_{3}\right) .
$$


Nous aurons alors, d'après le Lemme 9,

$$
\begin{aligned}
f(\sigma)-y_{3}^{-\sigma^{\prime}} f\left(\sigma-\sigma^{\prime}\right) & \gg \exp \left(-a_{4} t_{3} \log \left(1+t_{3}\right)\right) \frac{E_{1}\left(y_{3}\right)^{\ell}}{\ell !} \\
& \gg \exp \left(-a_{18} t \log (1+t)\right) \frac{E_{1}(y)^{\ell}}{\ell !}
\end{aligned}
$$

où $\sigma=2 \sigma^{\prime}=2 K\left(t_{3}+1\right) / \log y_{3}$, quitte à augmenter $a_{15}$ et $a_{16}$.

Pour conclure, il suffit de voir que

$$
\sum_{b \leq y_{3}, \Omega(b)=\ell} \frac{1}{b} \geq f(\sigma)-y_{3}^{-\sigma^{\prime}} f\left(\sigma-\sigma^{\prime}\right)
$$

En effet, si $b \leq y_{3}$ on a :

$$
1 \geq b^{-\sigma}-y_{3}^{-\sigma^{\prime}} b^{\sigma^{\prime}-\sigma}
$$

et si $b>y_{3}$, on a

$$
0 \geq b^{-\sigma}-y_{3}^{-\sigma^{\prime}} b^{\sigma^{\prime}-\sigma}
$$

LEMME 11. - En désignant par a un entier générique tel que $\Omega_{E}(a)=0$, on a uniformément pour $y \geq 1$ :

$$
\sum_{a \leq y} \frac{1}{a} \gg \log (2 y) e^{-E_{1}(\dot{y})} .
$$

Démonstration. - Ce résultat est classique. Désignons par $u$ un entier générique tel que :

$$
p \mid u \Longrightarrow p \in E \text {. }
$$

Tout entier $n \leq y$ s'écrit de manière unique $n=a u$. Donc :

$$
\begin{aligned}
\log (2 y) & \ll \sum_{n \leq y} \frac{1}{n} \\
& \leq \sum_{a \leq y} \frac{1}{a} \sum_{u \leq y} \frac{1}{u} \\
& \leq \sum_{a \leq y} \frac{1}{a} \prod_{p \in E}\left(1-\frac{1}{p}\right)^{-1} \\
& \ll \sum_{a \leq y} \frac{1}{a} \exp \left(E_{1}(y)\right) .
\end{aligned}
$$

TOME $117-1989-\mathrm{N}^{\mathrm{O}} 4$ 
LEMme 12. - On a uniformément pour $0 \leq \ell<p_{1} E_{1}(y)$ et $E_{1}(y) \geq a_{15} t \log (1+t)+a_{16}$, où $t=\ell / E_{1}(y)$ :

$$
\sum_{\substack{\psi(m) \leq y p_{1}^{-8} \\ \Omega_{E}(m)=\ell}} \frac{1}{\psi(m)} \gg \exp \left(-a_{14} t \log (1+t)-E_{1}(y)\right) \frac{\left(p_{1} E_{1}(y)\right)^{\ell}}{\ell !} \log y
$$

Démonstration. - Employons de nouveau la décomposition $m=a b$ :

$$
\begin{aligned}
\sum_{\substack{\psi(m) \leq y p_{1}^{-8} \\
\Omega_{E}(m)=\ell}} \frac{1}{\psi(m)} & =p_{1}^{\ell} \sum_{\substack{a b \leq p_{1}^{\ell-8} y \\
\Omega(b)=\ell}} \frac{1}{a b} \\
& \geq p_{1}^{\ell} \sum_{a \leq \sqrt{y}} \frac{1}{a} \sum_{\substack{b \leq \sqrt{y} p_{1}^{-8} \\
\Omega(b)=\ell}} \frac{1}{b} \\
& \gg p_{1}^{\ell}(\log y) e^{-E_{1}(y)} \exp \left(-a_{14} t \log (1+t)\right) \frac{E_{1}(y)^{\ell}}{\ell !}
\end{aligned}
$$

d'après les Lemmes 11,2 et 10.

Nous pouvons maintenant démontrer (19). Pour $y>1$, nous avons :

$$
\begin{gathered}
(\log y) C(y, \ell) \geq \sum_{\substack{\psi(n) \leq y \\
\Omega_{E}(n)=\ell}}^{*}\left\{\sum_{\substack{p \mid n \\
p \notin E}} \log p+\sum_{\substack{p \mid n \\
p \in E_{1}}} \log \frac{p}{p_{1}}\right\} \\
=\sum_{\substack{\psi(m) \leq y \\
\Omega_{E}(m)=\ell}}^{*} \sum_{\substack{p \leq y / \psi(m) \\
p \notin E}} \log p+\sum_{\substack{\psi(m) \leq y \\
\Omega_{E}(m)=\ell-1}} \sum_{\substack{p \leq p_{1} y / \psi(m) \\
p \in E_{1}}} \log \frac{p}{p_{1}}
\end{gathered}
$$

De même :

$$
\begin{aligned}
(\log y) C(y, \ell-1) \geq \sum_{\substack{\psi(m) \leq y \\
\Omega_{E}(m)=\ell-1}}^{*} \sum_{\substack{p \leq y / \psi(m) \\
p \notin E}} \log p \\
+\sum_{\substack{\psi(m) \leq y \\
\Omega_{E}(m)=\ell-2}}^{*} \sum_{\substack{p \leq p_{1} y / \psi(m) \\
p \in E_{1}}} \log \frac{p}{p_{1}}
\end{aligned}
$$

En ajoutant (38) et (39) et en ne gardant que les termes correspondant BULLETIN DE LA SOCIÉTÉ MATHÉMATIQUE DE FRANCE 
aux $m$ tels que $\Omega_{E}(m)=\ell-1$, on obtient :

$$
\begin{aligned}
\log y\{C(y, \ell)+C(y, \ell-1)\} \\
\geq \sum_{\substack{\psi(m) \leq y \\
\Omega_{E}(m)=\ell-1}}^{*}\left\{\sum_{\substack{p \leq y / \psi(m) \\
p \notin E}} \log p+\sum_{\substack{p \leq p_{1} y / \psi(m) \\
p \in E_{1}}} \log \frac{p}{p_{1}}\right\} \\
\geq \frac{1}{2} \sum_{\substack{\psi(m) \leq y p_{1}^{-8} \\
\Omega_{E}(m)=\ell-1}}^{*} \frac{y}{\psi(m)} \\
\gg e^{-a_{14} t \log (1+t)-E_{1}(y)} y \log y \frac{\left(p_{1} E_{1}(y)\right)^{\ell-1}}{(\ell-1) !}
\end{aligned}
$$

d'après les Lemmes 3 et 12, d'où le résultat.

\section{Démonstration du Corollaire 2}

La majoration de $P_{x}\left(\Omega_{E}(n) \geq k\right)$ contenue dans (13) étant une conséquence évidente de (12), il nous suffit de démontrer la minoration

$$
P_{x}\left(\Omega_{E}(n)=k\right) \gg p_{1}^{-k} \exp \left(-c_{14} p_{1} \log p_{1}+\left(p_{1}-1\right) E(x)\right) \varepsilon^{p_{1}-1}
$$

sous les hypothèses

$$
\begin{aligned}
& x>3, \quad(\log x)^{-1}<\varepsilon<1, \\
& E(x)>-\log \varepsilon+c_{12} p_{1} \log p_{1}, \\
& p_{1} E(x)-\left\{p_{1} E(x)\right\}^{1 / 2} \leq k \leq(1-\varepsilon) \frac{\log x}{\log p_{1}} .
\end{aligned}
$$

En posant $y=x p_{1}^{-k}$, on a donc $y \geq x^{\varepsilon}$. Par conséquent, d'après (21), on a

$$
\begin{aligned}
E_{1}(y) & \geq E_{1}\left(x^{\varepsilon}\right) \\
& \geq E_{1}(x)+\log \varepsilon+\mathrm{O}(1) \\
& \geq c_{12} p_{1} \log p_{1}+\mathrm{O}(1) \\
& \geq c_{8} t \log (1+t)+c_{9}
\end{aligned}
$$

si $c_{12}$ est assez grand, où $t=\min \left(p_{1}, k / E_{1}(y)\right)$.

D'autre part, la fonction $u \mapsto u-\sqrt{u}$ est croissante pour $u>\frac{1}{4}$. Donc, quitte à augmenter $c_{12}$,

$$
p_{1} E(x)-\left\{p_{1} E(x)\right\}^{1 / 2}>p_{1} E_{1}(y)-\left\{p_{1} E_{1}(y)\right\}^{1 / 2} .
$$

TOME $117-1989-\mathrm{N}^{\circ} 4$ 
D'après (9) et (24) on a donc :

$$
\begin{aligned}
P_{x}\left(\Omega_{E}(n) \geq k\right) & \geq P_{x}\left(\Omega_{E}(n)=k\right) \\
& \gg p_{1}^{-k} \exp \left(-c_{7} p_{1} \log \left(1+p_{1}\right)+\left(p_{1}-1\right) E_{1}(y)\right) \\
& \gg p_{1}^{-k} \exp \left(-c_{14} p_{1} \log p_{1}+\left(p_{1}-1\right) E(x)\right) \varepsilon^{p_{1}-1}
\end{aligned}
$$

ce qui démontre (40).

\section{Démonstration de (14)}

Pour démontrer (14) nous allons minorer $x P_{x}\left(\Omega_{E}(n)=k\right)$ dans un cas particulier. Soient $y$ et $z$ deux nombres premiers consécutifs et assez grands, $E$ l'ensemble des nombres premiers $\geq y$ (donc $p_{1}=y, p_{2}=z$ ) et $x=z y^{k}-1, k$ étant déterminé plus tard. On a $E_{1}\left(x / p_{1}^{k}\right)=0$. Soient $\gamma$ et $c$ des constantes absolues vérifiant :

$$
0<\gamma<1, \quad c>0 \quad \text { et } \quad \pi(u+v)-\pi(u) \geq \frac{c v}{\log v}+1
$$

pour tout $v \geq u^{\gamma}, u$ assez grand.

Tout entier $n=q_{1} \cdots q_{k}$ est compté dans $x P_{x}\left(\Omega_{E}(n)=k\right)$ si $q_{1}, \ldots, q_{k}$ sont des nombres premiers vérifiant :

$$
y \leq q_{1}<r y, \quad r y \leq q_{2}<r^{2} y, \ldots, r^{k-1} y \leq q_{k}<r^{k} y
$$

$r$ étant un réel $>1$ tel que :

$$
r^{k(k+1) / 2}=z
$$

On a $r=z^{2 / k(k+1)}$, donc $r-1 \geq 2 \log z / k(k+1)$. Définissons $k$ comme le plus grand entier tel que $2 \log z / k(k+1) \geq y^{-1+\gamma}$. On a donc pour tout $\ell=0, \ldots, k-1$

$$
\left(r^{\ell} y\right)^{1-\gamma}(r-1) \geq 1, \quad \text { puis } \quad r^{\ell+1} y-r^{\ell} y \geq\left(r^{\ell} y\right)^{\gamma} .
$$

On en déduit :

$$
\begin{aligned}
x P_{x}\left(\Omega_{E}(n)=k\right) & \geq(\pi(r y)-\pi(y)-1) \cdots\left(\pi\left(r^{k} y\right)-\pi\left(r^{k-1} y\right)-1\right) \\
& \geq c^{k} \frac{(y(r-1))(r y(r-1)) \cdots\left(r^{k-1} y(r-1)\right)}{\log (y(r-1)) \log (r y(r-1)) \cdots \log \left(r^{k-1} y(r-1)\right)} \\
& \geq\left(\frac{c(r-1) y r^{(k-1) / 2}}{\log (y(r-1))+(k-1) \log r}\right)^{k} .
\end{aligned}
$$

BULLETIN DE LA SOCIÉTÉ MATHÉMATIQUE DE FRANCE 
Quand $y$ tend vers l'infini, on a $z \sim y, 2(\log z) / k^{2} \sim y^{\gamma-1}$ donc $r-1 \sim \log r \sim y^{\gamma-1}$ et $(k-1) \log r=\mathrm{o}(1)$. Il en résulte que

$$
\frac{c(r-1) y r^{(k-1) / 2}}{\log (y(r-1))+(k-1) \log r} \sim \frac{c y^{\gamma}}{\gamma \log y} .
$$

Comme d'autre part, $x \sim y^{k+1}$, si $0<c^{\prime}<c / \gamma$ et $0<\lambda<1$, on a pour $y$ assez grand

$$
x P_{x}\left(\Omega_{E}(n)=k\right) \geq \lambda \frac{x}{y^{k+1}}\left(\frac{c^{\prime} y^{\gamma}}{\log y}\right)^{k} .
$$

Si $0<\gamma^{\prime}<\gamma$, pour $y$ assez grand cela est $\geq x / y^{k} y^{\gamma^{\prime} k}=x p_{1}^{-k} y^{\gamma^{\prime} k}$.

Comme $E_{1}(y)=0$ et comme $\gamma^{\prime} k \log y \sim \gamma^{\prime} \sqrt{2} y^{1-\gamma / 2}(\log y)^{3 / 2}$, cela démontre (14) avec $\tau=1-\gamma / 2$. La valeur de $\tau$ donnée dans (14) provient d'un résultat de C. J. Mozzochi ( $c f$. [11]) affirmant qu'on peut prendre $\gamma=11 / 20-1 / 406+\varepsilon$, où $\varepsilon$ est $>0$ quelconque.

\section{BIBLIOGRAPHIE}

[1] Balazard (M.). - Sur la répartition des valeurs de certaines fonctions arithmétiques additives, Thèse, Université de Limoges, 1987 .

[2] Balazard (M.), Delange (H.) et Nicolas (J.L.). - Sur le nombre de facteurs premiers des entiers, C.R. Acad. Sci. Paris Sér. I Math., t. 306, 1988, p. 511-514.

[3] Delange (H.). - A theorem on integral - valued additive functions, Illinois J. Math., t. 18, $\mathrm{n}^{\circ}$ 3, 1974, p. 357-372.

[4] Elliott (P.D.T.A.). - Probabilistic number theory, vol. 2. - New York, Heidelberg, Berlin, Springer-Verlag, 1979-1980.

[5] ERdös (P.) and Ruzsa (I.Z.). - On the small sieve, I : Sifting by primes, J. Number Theory, t. 12, 1980, p. 385-394.

[6] HalÁsz (G.). - On the distribution of additive and the mean values of multiplicative arithmetic functions, Studia Sci. Math. Hungar., t. 6, 1971 , p. $211-233$.

[7] HalÁsz (G.). - Remarks to my paper "On the distribution of additive and the mean values of multiplicative arithmetic functions", Studia Sci. Math. Hungar., t. 23, 1972, p. 425-432. 
[8] Hall (R.R.) and Tenenbaum (G.). - Divisors. - Cambridge University Press, 1988.

[9] Hildebrand (A.). - Quantitative mean value theorems for non negative multiplicative functions, II, Acta Arith., t. 48, 1987, p. 209260.

[10] Hildebrand (A.) and Tenenbaum (G.). - On the distribution of round numbers, Duke Math. J., t. 56, 1988, p. 471-501.

[11] Mozzochi (C.J.). - On the difference between consecutive primes, J. Number Theory, t. 24, 1986, p. 181-187.

[12] Nicolas (J.L.). - Sur la distribution des nombres entiers ayant une quantité fixée de facteurs premiers, Acta Arith., t. 44, 1984, p. 191200.

[13] Nicolas (J.L.). - Distribution des valeurs de la fonction d'Euler, Enseign. Math., t. 30, 1984, p. 331-338.

[14] NorTon (K.K.). - On the number of restricted prime factors of an integer, I, II, III, IV.

I : Illinois J. Math, t. 20, 1976, p. 681-705;

II : Acta Math., t. 143, 1979, p. 9-38;

III : Enseign. Math., t. 28, 1982, p. 31-52;

IV : Abstracts Amer. Math. Soc., t. 2, 1981, p. 366.

[15] Norton (K.K.). - Estimates for partial sums of the exponential series, J. Math. Analysis, t. 63, 1976, p. 265-296.

[16] Pomerance (C.). - On the distribution of round numbers, [in K. Alladi, Editeur], Number Theory, (Proc. Ootacamund, India), 1984, Springer LN, 1122 .

[17] Ramanujan (S.). - Collected Papers. - Chelsea Publishing Company, 1962.

[18] SÁrköZy (A.). - Remarks on a paper of G. Halăsz, Period. Math. Hungar., t. 8, 1977, p. 135-150.

[19] Selberg (A.). - Note on a paper by L. G. Sathe, J. Indian Math. Soc., t. 18, 1954, p. 83-87.

[20] Schoenfeld (L.). - Sharper Bounds for the Chebyshev Functions $\theta(x)$ and $\psi(x)$, II, Math. Comp., t. 30, 1976, p. 337-360. 